Mathematical Modelling and AnAlysis

Volume 17 Number 3, June 2012, 403-422

http://dx.doi.org/10.3846/13926292.2012.686067

(c) Vilnius Gediminas Technical University, 2012
Publisher: Taylor\&Francis and VGTU

http://www.tandfonline.com/TMMA

Print ISSN: 1392-6292

Online ISSN: 1648-3510

\title{
A Viscous Cahn-Hilliard Equation with Periodic Gradient Dependent Potentials and Sources
}

\author{
Yinghua $\mathrm{Li}^{a}$ and Yang $\mathrm{Cao}^{b}$ \\ ${ }^{a}$ School of Mathematical Sciences, South China Normal University \\ 510631 Guangzhou, China \\ ${ }^{b}$ School of Mathematical Sciences, Dalian University of Technology \\ 116024 Dalian, China \\ E-mail: yinghua@scnu.edu.cn \\ E-mail(corresp.): mathcy@dlut.edu.cn
}

Received April 3, 2011; revised March 13, 2012; published online June 1, 2012

Abstract. In this paper, we investigate the existence, uniqueness, attractivity and limiting process of solutions to the viscous Cahn-Hilliard equation with time periodic gradient dependent potentials and sources.

Keywords: viscous Cahn-Hilliard, periodicity, attractivity, limiting process.

AMS Subject Classification: 35B10; 35K55; 35K25.

\section{Introduction}

This paper is concerned with the following viscous Cahn-Hilliard equation in one spatial dimension

$$
\frac{\partial u}{\partial t}+\gamma D^{4} u-k \frac{\partial D^{2} u}{\partial t}=D \Phi(D u, t)-A(u, t)+f(x, t), \quad(x, t) \in Q
$$

subject to the boundary value conditions

$$
u(0, t)=u(1, t)=D^{2} u(0, t)=D^{2} u(1, t)=0, \quad t \geq 0,
$$

where $Q \equiv(0,1) \times \mathbb{R}^{+}, D=\partial / \partial x, \gamma>0$ denotes the mobility, $k>0$ is the viscous coefficient, $\Phi(s, t)=a(t)|s|^{\alpha-1} s-b(t) s$ and $A(s, t)=c(t)|s|^{\beta-1} s-d(t) s$ represent the potentials and the sources, respectively, $\alpha \geq 2, \beta>1, a(t), b(t)$, $c(t), d(t) \in C^{1+\nu}\left(\mathbb{R}^{+}\right)$are positive periodic functions with period $\omega>0$ and some $\nu \in(0,1)$, another source $f(x, t) \in C^{1+\nu, \nu / 4}(\bar{Q})$ is nontrivial and satisfies $f(x, t+\omega)=f(x, t)$ for all $(x, t) \in Q$. We will mainly investigate two kinds of problems in the present paper: $(1.1),(1.2)$ with the time periodic condition

$$
u(x, t+\omega)=u(x, t), \quad(x, t) \in Q,
$$


and (1.1), (1.2) with the initial value condition

$$
u(x, 0)=u_{0}(x), \quad x \in[0,1] .
$$

Equations (1.1) come from a variety of diffusion phenomena appeared widely in nature, including mathematical biology, fluid mechanics, etc. Note that if taking $k=0$, we obtain the Cahn-Hilliard equation with gradient dependent potentials and sources which was proposed by King [7] in 2003 by considering the height of a film in epitaxial growth of nanoscale thin films with $u(x, t)$ denoting the thickness of thin film. Further, when nonlocal effects and long range diffusion are taken into account, a similar model to (1.1) by considering the process of cell increasing with $u(x, t)$ representing the concentration or density of the cell was presented in [16]. When $k>0,(1.1)$ becomes the viscous Cahn-Hilliard equation and the viscous term describes the phenomenon of the speed of cell increasing slowing as a result of viscous relaxation effects, or viscosity. This type of equation was first propounded by Novick-Cohen [18] in 1988 to account for viscous effects in the phase separation of polymer-polymer systems.

In the past decades, the Cahn-Hilliard type equations with coefficients independent of $t$ have been deeply investigated, and there are a number of papers dealing with the theoretical and numerical analysis of existence of weak and classical solutions, stationary solutions, qualitative behavior, and practical finite element approximation $[1,4,5,6,10,13,14,17,20]$, etc. In particular, it has been shown in $[3,7,15]$ that all solutions of the initial boundary value problem converge to steady states as time tends to infinity. Since the viscous Cahn-Hilliard equation can describe the variation of cells that are sensitive to seasons, it is reasonable to study the problem with time periodic coefficients. However, just due to the appearance of the time periodic coefficients, it is of no meaning to consider the steady states. Hence, finding a new way to describe the asymptotic behavior of solutions of the initial boundary value problem seems much more interesting. In fact, it is shown in $[2,8,19,22]$ that all solutions may converge exponentially or globally to the set of periodic solutions as time tends to infinity. Specially, the results of $[19,22]$ imply that if one can find the maximal and minimal periodic solutions, then solutions of the initial and boundary value problems may be attracted globally to the sector between the maximal and minimal periodic solutions. Moreover, if the maximal and minimal periodic solutions are equivalent, then the unique periodic solution is a global attractor. However, those results are mostly for ODEs and second order parabolic equations.

The main purpose of this paper is devoted to discussing the viscous CahnHilliard equation with periodic gradient dependent potentials and sources (1.1), which has not been investigated extensively. After establishing the existence of time periodic solutions of the problem (1.1)-(1.3) and the existence and uniqueness of solutions to the initial boundary value problem (1.1), (1.2) and (1.4), we show that there exists an attractor which consists of the unique nontrivial time periodic solution of the periodic problem (1.1)-(1.3). Precisely speaking, any nontrivial solution of the initial boundary problem (1.1), (1.2) and (1.4) will be attracted into any small neighborhood of the attractor as time tends to infinity, 
which expresses that the effect of this unique time periodic solution is similar with that of the steady states solution of the initial boundary value problem with coefficients independent of $t$. This feature is different from that of the Cahn-Hilliard equation with time periodic concentration dependent potentials and sources

$$
\frac{\partial u}{\partial t}+\gamma D^{4} u-k \frac{\partial D^{2} u}{\partial t}=D^{2} \varphi(u, t)+f(x, t) .
$$

It has been only proved that the solutions to the initial boundary value problem of the above equation can be bounded by a suitable upper bound of the time periodic solutions for all large times, see [11, 21]. Moreover, for the CahnHilliard equation with gradient dependent potentials, our research also disclose that for the viscous case, the attractivity of periodic solution is under the $H^{1}$ norm, which is different from that of the nonviscous case, where the attractivity is under the $L^{2}$ norm [12].

Furthermore, we discuss the limiting process of time periodic solutions and the solutions of initial boundary value problems as the viscous coefficient $k$ tends to 0 (for the case $k=0$ we refer readers to [12]), and there is another difference between the characters of the solutions to the Cahn-Hilliard equations with periodic concentration dependent potentials and with periodic gradient dependent potentials. In fact, when the viscous coefficient $k$ tends to zero, for the case of concentration dependent potentials, the time periodic solutions and the solutions of the initial boundary value problem are almost everywhere convergent to the corresponding solutions of the problems with $k=0$ (see [11]), while for the case of gradient dependent potentials, the time periodic solutions and the solutions of the initial boundary value problem are uniformly convergent to the corresponding solutions of the problems with $k=0$ (see Theorem 4 and Theorem 5).

The structure of this paper is as follows. In Section 2, we show the existence of time periodic solutions to the problem (1.1)-(1.3). Then, we prove the existence and uniqueness of solutions to the initial boundary value problem (1.1), (1.2) and (1.4) in Section 3. At last, in Section 4 and Section 5, we discuss the attractivity and limiting process of solutions, respectively.

\section{Time Periodic Solutions}

In this section, we deal with the existence of time periodic solutions to the problem (1.1)-(1.3).

Theorem 1. The problem (1.1)-(1.3) admits a nontrivial time periodic solution.

Proof. We are going to apply the Leray-Schauder fixed point theorem to prove this theorem. For this purpose, we consider a family of relevant equations with parameter, namely

$$
\frac{\partial u}{\partial t}+\gamma D^{4} u-k \frac{\partial D^{2} u}{\partial t}=D \Phi(D u, t)-\sigma A(u, t)+\sigma f(x, t), \quad(x, t) \in Q_{\omega}
$$


subject to the conditions

$$
\begin{aligned}
u(0, t) & =u(1, t)=D^{2} u(0, t)=D^{2} u(1, t)=0, \quad t \in[0, \omega], \\
u(x, 0) & =u(x, \omega), \quad x \in[0,1],
\end{aligned}
$$

where $\sigma \in[0,1]$ is a parameter, $Q_{\omega}=(0,1) \times(0, \omega)$.

We first give some necessary a priori estimates. Here and below, we denote by $C$ a constant, whose value may be different from line to line and is independent of $u$ and $\sigma$. Multiplying (2.1) by $u$ and integrating over $Q_{\omega}$, we have

$$
\begin{aligned}
& \gamma \iint_{Q_{\omega}}\left|D^{2} u\right|^{2} d x d t+\iint_{Q_{\omega}} a(t)|D u|^{\alpha+1} d x d t+\sigma \iint_{Q_{\omega}} c(t)|u|^{\beta+1} d x d t \\
& \quad=\iint_{Q_{\omega}} b(t)|D u|^{2} d x d t+\sigma \iint_{Q_{\omega}} d(t)|u|^{2} d x d t+\sigma \iint_{Q_{\omega}} f(x, t) u d x d t \\
& \quad \leq \frac{a}{2} \iint_{Q_{\omega}}|D u|^{\alpha+1} d x d t+\frac{c}{2} \sigma \iint_{Q_{\omega}}|u|^{\beta+1} d x d t+C,
\end{aligned}
$$

where $\underline{a}$ and $\underline{c}$ are the lower bounds of $a(t)$ and $c(t)$, respectively, which implies that

$$
\iint_{Q_{\omega}}\left|D^{2} u\right|^{2} d x d t \leq C, \iint_{Q_{\omega}}|D u|^{\alpha+1} d x d t \leq C, \sigma \iint_{Q_{\omega}}|u|^{\beta+1} d x d t \leq C .
$$

We multiply $(2.1)$ by $u_{t}$ and integrate the result with respect to $x$ over $(0,1)$ and obtain

$$
\begin{aligned}
\int_{0}^{1}\left|u_{t}\right|^{2} d x+\frac{\gamma}{2} \frac{\mathrm{d}}{\mathrm{d} t} \int_{0}^{1}\left|D^{2} u\right|^{2} d x+k \int_{0}^{1}\left|D u_{t}\right|^{2} d x \\
\quad+\frac{\mathrm{d}}{\mathrm{d} t} \int_{0}^{1} \Psi(D u, t) d x+\sigma \frac{\mathrm{d}}{\mathrm{d} t} \int_{0}^{1} B(u, t) d x \\
=\int_{0}^{1} \frac{a^{\prime}(t)}{\alpha+1}|D u|^{\alpha+1} d x-\int_{0}^{1} \frac{b^{\prime}(t)}{2}|D u|^{2} d x+\sigma \int_{0}^{1} \frac{c^{\prime}(t)}{\beta+1}|u|^{\beta+1} d x \\
\quad-\sigma \int_{0}^{1} \frac{d^{\prime}(t)}{2}|u|^{2} d x+\sigma \int_{0}^{1} f(x, t) u_{t} d x \\
\leq \int_{0}^{1} \frac{a^{\prime}(t)}{\alpha+1}|D u|^{\alpha+1} d x-\int_{0}^{1} \frac{b^{\prime}(t)}{2}|D u|^{2} d x+\sigma \int_{0}^{1} \frac{c^{\prime}(t)}{\beta+1}|u|^{\beta+1} d x \\
\quad-\sigma \int_{0}^{1} \frac{d^{\prime}(t)}{2}|u|^{2} d x+\frac{1}{2} \int_{0}^{1}\left|u_{t}\right|^{2} d x+C,
\end{aligned}
$$

where

$$
\Psi(s, t)=\frac{a(t)}{\alpha+1}|s|^{\alpha+1}-\frac{b(t)}{2} s^{2}, \quad B(s, t)=\frac{c(t)}{\beta+1}|s|^{\beta+1}-\frac{d(t)}{2} s^{2} .
$$


It follows that

$$
\begin{aligned}
& \frac{\mathrm{d}}{\mathrm{d} t} \int_{0}^{1}\left(\frac{\gamma}{2}\left|D^{2} u\right|^{2}+\Psi(D u, t)+\sigma B(u, t)\right) d x \\
& \quad \leq \int_{0}^{1}\left(\frac{a^{\prime}(t)}{\alpha+1}|D u|^{\alpha+1}-\frac{b^{\prime}(t)}{2}|D u|^{2}+\frac{c^{\prime}(t)}{\beta+1} \sigma|u|^{\beta+1}-\frac{d^{\prime}(t)}{2} \sigma|u|^{2}\right) d x+C, \\
& \quad t \in(0, \omega) .
\end{aligned}
$$

Furthermore, by the periodicity of the solution $u$ and (2.4), we obtain

$$
\iint_{Q_{\omega}}\left|u_{t}\right|^{2} d x d t \leq C, \quad \iint_{Q_{\omega}}\left|D u_{t}\right|^{2} d x d t \leq C .
$$

For all $t \in[0, \omega]$, set

$$
\begin{aligned}
& F(t)=\int_{0}^{1}\left(\frac{\gamma}{2}\left|D^{2} u\right|^{2}+\Psi(D u, t)+\sigma B(u, t)\right) d x \\
& =\int_{0}^{1}\left(\frac{\gamma}{2}\left|D^{2} u\right|^{2}+\frac{a(t)}{\alpha+1}|D u|^{\alpha+1}-\frac{b(t)}{2}|D u|^{2}+\frac{c(t)}{\beta+1} \sigma|u|^{\beta+1}-\frac{d(t)}{2} \sigma|u|^{2}\right) d x .
\end{aligned}
$$

From (2.6) we have

$$
\frac{d F}{d t} \leq C F(t)+C, \quad \forall t \in(0, \omega)
$$

On the other hand, by (2.4), we get

$$
\begin{aligned}
\int_{0}^{\omega} F(t) d t & \leq \frac{\gamma}{2} \iint_{Q_{\omega}}\left|D^{2} u\right|^{2} d x d t+C \iint_{Q_{\omega}}|D u|^{\alpha+1} d x d t+C \sigma \iint_{Q_{\omega}}|u|^{\beta+1} d x d t \\
& \leq C .
\end{aligned}
$$

Hence, there exists a point $\tilde{t} \in(0, \omega)$ such that

$$
F(\tilde{t})=\frac{1}{\omega} \int_{0}^{\omega} F(t) d t \leq C .
$$

For any $t \in(\tilde{t}, \omega]$, integrating $(2.8)$ from $\tilde{t}$ to $t$ yields

$$
F(t) \leq F(\tilde{t})+C \int_{0}^{\omega} F(t) d t+C \omega \leq C .
$$

Recalling the periodicity of $u$ again, we see that $F(0)=F(\omega) \leq C$. Thus, we get

$$
F(t) \leq F(0)+C \int_{0}^{\omega} F(t) d t+C \omega \leq C, \quad \forall t \in[0, \omega],
$$

from which and the definition of $F(t)$, we obtain

$$
\frac{\gamma}{2} \int_{0}^{1}\left|D^{2} u\right|^{2} d x+\frac{a(t)}{\alpha+1} \int_{0}^{1}|D u|^{\alpha+1} d x+\frac{c(t)}{\beta+1} \sigma \int_{0}^{1}|u|^{\beta+1} d x
$$




$$
\begin{aligned}
& \leq \frac{b(t)}{2} \int_{0}^{1}|D u|^{2} d x+\frac{d(t)}{2} \sigma \int_{0}^{1}|u|^{2} d x+C \\
& \leq \frac{\underline{a}}{2(\alpha+1)} \int_{0}^{1}|D u|^{\alpha+1} d x+\frac{\underline{c}}{2(\beta+1)} \sigma \int_{0}^{1}|u|^{\beta+1} d x+C .
\end{aligned}
$$

Therefore,

$$
\int_{0}^{1}|D u(x, t)|^{\alpha+1} d x \leq C, \quad \int_{0}^{1}\left|D^{2} u(x, t)\right|^{2} d x \leq C, \quad \forall t \in[0, \omega] .
$$

From the first inequality (2.9) and the boundary value conditions (2.2), we arrive at

$$
\|u\|_{L^{\infty}\left(\bar{Q}_{\omega}\right)} \leq C .
$$

Combining both inequalities (2.9), we can also deduce that

$$
\|D u\|_{L^{\infty}\left(\bar{Q}_{\omega}\right)} \leq C .
$$

Multiplying (2.1) by $D^{4} u$ and integrating the result with respect to $x$ over $(0,1)$ yield

$$
\begin{aligned}
& \frac{1}{2} \frac{\mathrm{d}}{\mathrm{d} t} \int_{0}^{1}\left(\left|D^{2} u\right|^{2}+k\left|D^{3} u\right|^{2}\right) d x+\gamma \int_{0}^{1}\left|D^{4} u\right|^{2} d x \\
& \quad=\int_{0}^{1} \Phi_{s}^{\prime}(D u, t) D^{2} u D^{4} u d x-\sigma \int_{0}^{1} A(u, t) D^{4} u d x+\sigma \int_{0}^{1} f(x, t) D^{4} u d x \\
& \quad \leq \frac{\gamma}{2} \int_{0}^{1}\left|D^{4} u\right|^{2} d x+C \int_{0}^{1}\left|D^{2} u\right|^{2} d x+C .
\end{aligned}
$$

From (2.9), we have

$$
\frac{\mathrm{d}}{\mathrm{d} t} \int_{0}^{1}\left(\left|D^{2} u\right|^{2}+k\left|D^{3} u\right|^{2}\right) d x \leq C, \quad \forall t \in(0, \omega) .
$$

Moreover, by the periodicity of $u$, we get

$$
\iint_{Q_{\omega}}\left|D^{4} u\right|^{2} d x d t \leq C
$$

Consequently,

$$
\begin{aligned}
\iint_{Q_{\omega}}\left|D^{3} u\right|^{2} d x d t & =-\iint_{Q_{\omega}} D^{2} u D^{4} u d x d t \\
& \leq \frac{1}{2} \iint_{Q_{\omega}}\left(\left|D^{2} u\right|^{2}+\left|D^{4} u\right|^{2}\right) d x d t \leq C .
\end{aligned}
$$

Combining (2.12) with the first inequality of (2.4) and (2.14) gives

$$
\int_{0}^{1}\left|D^{3} u(x, t)\right|^{2} d x \leq C, \quad \forall t \in[0, \omega] .
$$


From (2.9) and (2.15), we have

$$
\left\|D^{2} u\right\|_{L^{\infty}\left(\bar{Q}_{\omega}\right)} \leq C .
$$

We rewrite the equation (2.1) into the following form

$$
D^{2} u_{t}=\frac{1}{k} u_{t}+\frac{\gamma}{k} D^{4} u-\frac{1}{k} D \Phi(D u, t)+\frac{\sigma}{k} A(u, t)-\frac{\sigma}{k} f(x, t) .
$$

From (2.7), (2.10), (2.11), (2.13) and (2.16), we obtain

$$
\iint_{Q_{\omega}}\left|D^{2} u_{t}\right|^{2} d x d t \leq C
$$

In the following, we give Hölder's norm estimates of $u$. By virtue of (2.11), we see that

$$
\left|u\left(x_{1}, t\right)-u\left(x_{2}, t\right)\right| \leq C\left|x_{1}-x_{2}\right|, \quad \forall t \in[0, \omega], x_{1}, x_{2} \in[0,1] .
$$

For any $\left(x, t_{1}\right),\left(x, t_{2}\right) \in \bar{Q}_{\omega}$, consider the case of $0 \leq x \leq 1 / 2$. Suppose that $\Delta t=t_{2}-t_{1}>0$ satisfying $(\Delta t)^{1 / 2} \leq 1 / 4$. Integrating (2.1) over $(y, y+$ $\left.(\Delta t)^{1 / 2}\right) \times\left(t_{1}, t_{2}\right)$ yields

$$
\begin{aligned}
& \int_{y}^{y+(\Delta t)^{1 / 2}}\left(u\left(z, t_{2}\right)-u\left(z, t_{1}\right)\right) d z=-\gamma \int_{t_{1}}^{t_{2}} \int_{y}^{y+(\Delta t)^{1 / 2}} D^{4} u d z d t \\
& +k \int_{t_{1}}^{t_{2}} \int_{y}^{y+(\Delta t)^{1 / 2}} D^{2} u_{t} d z d t+\int_{t_{1}}^{t_{2}} \int_{y}^{y+(\Delta t)^{1 / 2}} D \Phi(D u, t) d z d t \\
& \quad-\sigma \int_{t_{1}}^{t_{2}} \int_{y}^{y+(\Delta t)^{1 / 2}}(A(u, t)-f(z, t)) d z d t
\end{aligned}
$$

Integrating the above equality with respect to $y$ over $\left(x, x+(\Delta t)^{1 / 2}\right)$, we arrive at

$$
\left|u\left(x^{*}, t_{2}\right)-u\left(x^{*}, t_{1}\right)\right| \leq C\left|t_{2}-t_{1}\right|^{1 / 4}
$$

where $x^{*}=y^{*}+\theta^{*}(\Delta t)^{1 / 2}, y^{*} \in\left(x, x+(\Delta t)^{1 / 2}\right), \theta^{*} \in(0,1)$. Combining the above inequality with $(2.18)$ gives

$$
\begin{aligned}
\left|u\left(x, t_{1}\right)-u\left(x, t_{2}\right)\right| \leq & \left|u\left(x, t_{1}\right)-u\left(x^{*}, t_{1}\right)\right|+\left|u\left(x^{*}, t_{1}\right)-u\left(x^{*}, t_{2}\right)\right| \\
& +\left|u\left(x, t_{2}\right)-u\left(x^{*}, t_{2}\right)\right| \leq C\left|t_{1}-t_{2}\right|^{1 / 4} .
\end{aligned}
$$

Hence,

$$
\left|u\left(x_{1}, t_{1}\right)-u\left(x_{2}, t_{2}\right)\right| \leq C\left(\left|x_{1}-x_{2}\right|+\left|t_{1}-t_{2}\right|^{1 / 4}\right)
$$

for all $\left(x_{i}, t_{i}\right) \in \bar{Q}_{\omega}(i=1,2)$. Next, we claim that

$$
\left|D u\left(x_{1}, t_{1}\right)-D u\left(x_{2}, t_{2}\right)\right| \leq C\left(\left|x_{1}-x_{2}\right|+\left|t_{1}-t_{2}\right|^{1 / 2}\right)
$$

for all $\left(x_{i}, t_{i}\right) \in \bar{Q}_{\omega}(i=1,2)$. Obviously, the above inequality is equivalent to

$$
\begin{aligned}
& \left|D u\left(x_{1}, t\right)-D u\left(x_{2}, t\right)\right| \leq C\left|x_{1}-x_{2}\right|, \quad \forall t \in[0, \omega], x_{1}, x_{2} \in[0,1], \\
& \left|D u\left(x, t_{1}\right)-D u\left(x, t_{2}\right)\right| \leq C\left|t_{1}-t_{2}\right|^{1 / 2}, \quad \forall x \in[0,1], t_{1}, t_{2} \in[0, \omega] .
\end{aligned}
$$


In fact, (2.21) is a direct consequence of (2.16). Recalling the second inequality (2.7) and by using the mean value theorem, we see that there exists a point $\tilde{x} \in(0,1)$ such that

$$
\int_{0}^{\omega}\left|\frac{\partial D u}{\partial t}(\tilde{x}, t)\right|^{2} \mathrm{~d} t \leq C .
$$

Integrating the equation $(2.1)$ over $(\tilde{x}, x) \times\left(t_{1}, t_{2}\right)$, we have

$$
\begin{aligned}
& k\left(D u\left(x, t_{2}\right)-D u\left(x, t_{1}\right)\right)=k \int_{t_{1}}^{t_{2}} \frac{\partial D u}{\partial t}(\tilde{x}, t) d t+\int_{\tilde{x}}^{x} \int_{t_{1}}^{t_{2}} \frac{\partial u}{\partial t}(z, t) d t d z \\
& +\gamma \int_{\tilde{x}}^{x} \int_{t_{1}}^{t_{2}} D^{4} u(z, t) d t d z-\int_{\tilde{x}}^{x} \int_{t_{1}}^{t_{2}} \Phi_{s}^{\prime}(D u(z, t), t) D^{2} u(z, t) d t d z \\
& \quad+\sigma \int_{\tilde{x}}^{x} \int_{t_{1}}^{t_{2}} A(u(z, t), t) d t d z-\sigma \int_{\tilde{x}}^{x} \int_{t_{1}}^{t_{2}} f(z, t) d t d z .
\end{aligned}
$$

Integrating the above equality with respect to $x$ over $\left(y, y+(\Delta t)^{1 / 2}\right)$, we obtain

$$
\left|D u\left(x^{*}, t_{2}\right)-D u\left(x^{*}, t_{1}\right)\right| \leq C(\Delta t)^{1 / 2},
$$

where $x^{*} \in\left(y, y+(\Delta t)^{1 / 2}\right)$. Combining the above inequality with (2.21) yields

$$
\left|D u\left(x, t_{1}\right)-D u\left(x, t_{2}\right)\right| \leq C\left|t_{1}-t_{2}\right|^{1 / 2} .
$$

Now, we define an operator $F$ on linear space $X$ as follows

$$
F: X \longrightarrow X, \quad u \longmapsto w,
$$

where

$$
X=\left\{u \in C^{2,1 / 4}\left(\bar{Q}_{\omega}\right) ; u(0, t)=u(1, t)=0, \forall t \in[0, \omega]\right\},
$$

and $w$ is a solution of the following problem

$$
\begin{aligned}
& \frac{\partial w}{\partial t}+\gamma D^{4} w-k \frac{\partial D^{2} w}{\partial t}=\Phi_{s}^{\prime}(D u, t) D^{2} w-A(u, t)+f(x, t), \quad(x, t) \in Q_{\omega}, \\
& w(0, t)=w(1, t)=D^{2} w(0, t)=D^{2} w(1, t)=0, \quad t \in[0, \omega], \\
& w(x, 0)=w(x, \omega), \quad x \in[0,1],
\end{aligned}
$$

where $\Phi_{s}^{\prime}(s, t)=a(t) \alpha|s|^{\alpha-1}-b(t)$. From (2.19), (2.20) and the assumptions on $a(t), b(t), c(t), d(t), f(x, t)$ we see that $\Phi^{\prime}(D u, t) \in C^{\nu, \nu / 4}\left(\bar{Q}_{\omega}\right), A(u, t) \in$ $C^{1+\nu, \nu / 4}\left(\bar{Q}_{\omega}\right), f(x, t) \in C^{1+\nu, \nu / 4}\left(\bar{Q}_{\omega}\right)$. By the classical linear theory (see [9]), we know that the above problem admits a unique solution $w \in C^{4+\nu, 1+\nu / 4}\left(\bar{Q}_{\omega}\right)$, $D^{2} w_{t} \in C^{\nu, \nu / 4}\left(\bar{Q}_{\omega}\right)$. Hence, the operator $F$ is well-defined. We can also obtain the compactness of operator $F$ by means of the compact embedding theorem (see Lemma 2.1 in [23] for example). Moreover, if $u=\sigma F u$ for some $\sigma \in[0,1]$, then $u$ satisfies (2.1)-(2.3). Clearly $\sigma=0$ implies that $u \equiv 0$. If $\sigma \neq 0$, then from the above argument, we see that $\|u\|_{C^{2,1 / 4}\left(\bar{Q}_{\omega}\right)}$ is bounded and independent of $u$ and $\sigma$. Therefore, the Leray-Schauder fixed point theorem implies that the operator $F$ has a fixed point $u$, which is the desired time periodic solution of the problem (1.1)-(1.3). The proof of this theorem is complete. 


\section{Initial Boundary Value Problems}

After establishing the existence of time periodic solutions, we turn to the discussion of the solvability of the initial boundary value problem (1.1), (1.2) and (1.4).

Theorem 2. For any given initial datum $u_{0} \in C^{4+\alpha}[0,1]$ with $u_{0}(0)=u_{0}(1)=$ $D^{2} u_{0}(0)=D^{2} u_{0}(1)=0$, the initial boundary value problem (1.1), (1.2) and (1.4) admits a unique global classical solution.

Proof. To prove the existence of solutions to the problem (1.1), (1.2) and (1.4), we employ the Leray-Schauder fixed point theorem which enables us to study the problem by considering the following problem

$$
\begin{aligned}
& \frac{\partial u}{\partial t}+\gamma D^{4} u-k \frac{\partial D^{2} u}{\partial t}-D \Phi(D u, t)+\sigma A(u, t)=\sigma f(x, t), \quad(x, t) \in Q_{T}, \\
& u(0, t)=u(1, t)=D^{2} u(0, t)=D^{2} u(1, t)=0, \quad t \in[0, T], \\
& u(x, 0)=\sigma u_{0}(x), \quad x \in[0,1]
\end{aligned}
$$

where the parameter $\sigma \in[0,1], T>0$ is a given constant, $Q_{T}=(0,1) \times(0, T)$.

We first do some a priori estimates. Multiplying (3.1) by $u$ and integrating the result with respect to $x$ over $(0,1)$, we have

$$
\begin{aligned}
\frac{1}{2} \frac{\mathrm{d}}{\mathrm{d} t} & \int_{0}^{1} u^{2} d x+\gamma \int_{0}^{1}\left|D^{2} u\right|^{2} d x+\frac{k}{2} \frac{\mathrm{d}}{\mathrm{d} t} \int_{0}^{1}|D u|^{2} d x \\
& +\int_{0}^{1} a(t)|D u|^{\alpha+1} d x+\sigma \int_{0}^{1} c(t)|u|^{\beta+1} d x \\
= & \int_{0}^{1} b(t)|D u|^{2} d x+\sigma \int_{0}^{1} d(t) u^{2} d x+\sigma \int_{0}^{1} f(x, t) u d x \\
\leq & \frac{\gamma}{2} \int_{0}^{1}\left|D^{2} u\right|^{2} d x+C \int_{0}^{1} u^{2} d x+C .
\end{aligned}
$$

It follows that

$$
\frac{\mathrm{d}}{\mathrm{d} t} \int_{0}^{1}\left(u^{2}+k|D u|^{2}\right) d x \leq C \int_{0}^{1}\left(u^{2}+k|D u|^{2}\right) d x+C, \quad \forall t \in(0, T) .
$$

Then, using Gronwall's inequality gives

$$
\int_{0}^{1}\left(u^{2}(x, t)+k|D u(x, t)|^{2}\right) d x \leq C, \quad \forall t \in(0, T),
$$

from which we have

$$
\int_{0}^{1}|D u(x, t)|^{2} d x \leq C, \quad \forall t \in(0, T) .
$$

Recalling the boundary value conditions (3.2), we obtain

$$
\|u\|_{L^{\infty}\left(\bar{Q}_{T}\right)} \leq C .
$$


Moreover, integrating (3.4) from 0 to $T$ and using (3.6), we get

$$
\iint_{Q_{T}}\left|D^{2} u\right|^{2} d x d t \leq C, \quad \iint_{Q_{T}}|D u|^{\alpha+1} d x d t \leq C .
$$

We multiply (3.1) by $u_{t}$, integrate the result over $Q_{t}$ and obtain

$$
\begin{aligned}
& \iint_{Q_{t}}\left|u_{t}\right|^{2} d x d \tau+\frac{\gamma}{2} \int_{0}^{1}\left|D^{2} u(x, t)\right|^{2} d x+k \iint_{Q_{t}}\left|D u_{t}\right|^{2} d x d \tau \\
& \quad+\int_{0}^{1} \Psi(D u(x, t), t) d x+\sigma \int_{0}^{1} B(u(x, t), t) d x \\
& =\frac{\gamma \sigma^{2}}{2} \int_{0}^{1}\left|D^{2} u_{0}(x)\right|^{2} d x+\int_{0}^{1} \Psi\left(\sigma D u_{0}(x), 0\right) d x+\sigma \int_{0}^{1} B\left(\sigma u_{0}(x), 0\right) d x \\
& \quad+\iint_{Q_{t}} \frac{a^{\prime}(\tau)}{\alpha+1}|D u|^{\alpha+1} d x d \tau-\iint_{Q_{t}} \frac{b^{\prime}(\tau)}{2}|D u|^{2} d x d \tau \\
& \quad+\sigma \iint_{Q_{t}} \frac{c^{\prime}(\tau)}{\beta+1}|u|^{\beta+1} d x d \tau-\sigma \iint_{Q_{t}} \frac{d^{\prime}(\tau)}{2} u^{2} d x d \tau+\sigma \iint_{Q_{t}} f(x, \tau) u_{t} d x d \tau \\
& \leq \frac{1}{2} \iint_{Q_{t}}\left|u_{t}\right|^{2} d x d \tau+C,
\end{aligned}
$$

where $\Psi(s, t)$ and $B(s, t)$ are as in (2.5). Then, we have

$$
\begin{aligned}
\int_{0}^{1}\left|D^{2} u(x, t)\right|^{2} d x & \leq C, \quad \forall t \in(0, T), \\
\iint_{Q_{T}}\left|u_{t}\right|^{2} d x d t & \leq C, \quad \iint_{Q_{T}}\left|D u_{t}\right|^{2} d x d t \leq C .
\end{aligned}
$$

Combining (3.5) with (3.8), we arrive at

$$
\|D u\|_{L^{\infty}\left(\bar{Q}_{T}\right)} \leq C .
$$

Multiplying (3.1) by $D^{4} u$ and integrating over $Q_{t}$ yield

$$
\begin{aligned}
& \frac{1}{2} \int_{0}^{1}\left|D^{2} u(x, t)\right|^{2} d x+\gamma \iint_{Q_{t}}\left|D^{4} u\right|^{2} d x d \tau+\frac{k}{2} \int_{0}^{1}\left|D^{3} u(x, t)\right|^{2} d x \\
& =\frac{\sigma^{2}}{2} \int_{0}^{1}\left|D^{2} u_{0}(x)\right|^{2} d x+\frac{k \sigma^{2}}{2} \int_{0}^{1}\left|D^{3} u_{0}(x)\right|^{2} d x+\iint_{Q_{t}} \Phi_{s}^{\prime}(D u, \tau) D^{2} u D^{4} u d x d \tau \\
& \quad-\sigma \iint_{Q_{t}} A(u, \tau) D^{4} u d x d \tau+\sigma \iint_{Q_{t}} f(x, \tau) D^{4} u d x d \tau \\
& \leq \frac{\gamma}{2} \iint_{Q_{t}}\left|D^{4} u\right|^{2} d x d \tau+C,
\end{aligned}
$$

from which we have

$$
\int_{0}^{1}\left|D^{3} u(x, t)\right|^{2} d x \leq C, \quad \forall t \in(0, T), \quad \iint_{Q_{T}}\left|D^{4} u\right|^{2} d x d t \leq C .
$$


By (3.8) and (3.11), we obtain

$$
\left\|D^{2} u\right\|_{L^{\infty}\left(\bar{Q}_{T}\right)} \leq C .
$$

We rewrite the equation (3.1) into the following form

$$
D^{2} u_{t}=\frac{1}{k} u_{t}+\frac{\gamma}{k} D^{4} u-\frac{1}{k} D \Phi(D u, t)+\frac{\sigma}{k} A(u, t)-\frac{\sigma}{k} f(x, t) .
$$

From (3.6), (3.9), (3.10) and (3.12), we get

$$
\iint_{Q_{T}}\left|D^{2} u_{t}\right|^{2} d x d t \leq C .
$$

Similar to the proof of Theorem 1, it is easy to obtain

$$
\begin{aligned}
\left|u\left(x_{1}, t_{1}\right)-u\left(x_{2}, t_{2}\right)\right| & \leq C\left(\left|x_{1}-x_{2}\right|+\left|t_{1}-t_{2}\right|^{1 / 4}\right), \\
\left|D u\left(x_{1}, t_{1}\right)-D u\left(x_{2}, t_{2}\right)\right| & \leq C\left(\left|x_{1}-x_{2}\right|+\left|t_{1}-t_{2}\right|^{1 / 4}\right)
\end{aligned}
$$

for all $\left(x_{i}, t_{i}\right) \in \bar{Q}_{T}(i=1,2)$. Define a linear space

$$
X=\left\{u \in C^{2,1 / 4}\left(\bar{Q}_{T}\right) ; u(0, t)=u(1, t)=0, \forall t \in[0, T]\right\}
$$

and an operator $F$ by $F: X \longrightarrow X, \quad u \longmapsto w$, where $w$ is a solution of the following problem

$$
\begin{aligned}
& \frac{\partial w}{\partial t}+\gamma D^{4} w-k \frac{\partial D^{2} w}{\partial t}=\Phi_{s}^{\prime}(D u, t) D^{2} w-A(u, t)+f(x, t), \quad(x, t) \in Q_{T}, \\
& w(0, t)=w(1, t)=D^{2} w(0, t)=D^{2} w(1, t)=0, \quad t \in[0, T], \\
& w(x, 0)=u_{0}(x), \quad x \in[0,1]
\end{aligned}
$$

where $\Phi_{s}^{\prime}(s, t)=a(t) \alpha|s|^{\alpha-1}-b(t)$. From (3.14), (3.15) and the assumptions on $a(t), b(t), c(t), d(t), f(x, t)$ we know that $\Phi^{\prime}(D u, t) \in C^{\nu, \nu / 4}\left(\bar{Q}_{T}\right), A(u, t) \in$ $C^{1+\nu, \nu / 4}\left(\bar{Q}_{T}\right), f(x, t) \in C^{1+\nu, \nu / 4}\left(\bar{Q}_{T}\right)$. By the similar discussion in Theorem 1 , we can prove that the problem $(1.1),(1.2)$ and (1.4) admits a classical solution in $Q_{T}$. Then, we consider the problem in $Q_{(T, 2 T)}, Q_{(2 T, 3 T)}, \ldots, Q_{((n-1) T, n T)}, \ldots$ in turn. Finally, we know that the initial boundary value problem (1.1), (1.2) and (1.4) admits a classical solution in $Q$.

In what follows, we show the uniqueness of solutions. Let $u_{1}, u_{2}$ be two solutions of the problem (1.1), (1.2) and (1.4). Set $v=u_{1}-u_{2}$, then we have

$$
\begin{aligned}
\frac{1}{2} \frac{\mathrm{d}}{\mathrm{d} t} & \int_{0}^{1} v^{2} d x+\gamma \int_{0}^{1}\left|D^{2} v\right|^{2} d x+\frac{k}{2} \frac{\mathrm{d}}{\mathrm{d} t} \int_{0}^{1}|D v|^{2} d x \\
& +\int_{0}^{1} a(t)\left(\left|D u_{1}\right|^{\alpha-1} D u_{1}-\left|D u_{2}\right|^{\alpha-1} D u_{2}\right)\left(D u_{1}-D u_{2}\right) d x \\
& +\int_{0}^{1} c(t)\left(\left|u_{1}\right|^{\beta-1} u_{1}-\left|u_{2}\right|^{\beta-1} u_{2}\right)\left(u_{1}-u_{2}\right) d x \\
= & \int_{0}^{1} b(t)|D v|^{2} d x+\int_{0}^{1} d(t) v^{2} d x .
\end{aligned}
$$


Noticing that the constant $\gamma>0, \alpha \geq 2, \beta>1$ and $a(t), c(t)$ are positive functions, we see that

$$
\begin{aligned}
& \gamma \int_{0}^{1}\left|D^{2} v\right|^{2} d x+\int_{0}^{1} a(t)\left(\left|D u_{1}\right|^{\alpha-1} D u_{1}-\left|D u_{2}\right|^{\alpha-1} D u_{2}\right)\left(D u_{1}-D u_{2}\right) d x \\
& \quad+\int_{0}^{1} c(t)\left(\left|u_{1}\right|^{\beta-1} u_{1}-\left|u_{2}\right|^{\beta-1} u_{2}\right)\left(u_{1}-u_{2}\right) d x \geq 0 .
\end{aligned}
$$

Recalling the continuities of $b(t)$ and $d(t)$, we get

$$
\begin{aligned}
\frac{\mathrm{d}}{\mathrm{d} t} \int_{0}^{1}\left(v^{2}+k|D v|^{2}\right) d x & \leq 2 \int_{0}^{1} b(t)|D v|^{2} d x+2 \int_{0}^{1} d(t) v^{2} d x \\
& \leq C \int_{0}^{1}\left(v^{2}+k|D v|^{2}\right) d x, \quad \forall t \in(0, T) .
\end{aligned}
$$

By using Gronwall's inequality, we have

$$
\int_{0}^{1}\left(v^{2}(x, t)+k|D v(x, t)|^{2}\right) d x=0, \quad \forall t \in(0, T) .
$$

Hence, $\int_{0}^{1} v^{2}(x, t) d x=0, \forall t \in(0, T)$, which means that $u_{1}=u_{2}$ a.e. in $Q_{T}$. By virtue of the continuities of $u_{1}$ and $u_{2}$, we have $u_{1}=u_{2}$ in $Q_{T}$. The proof of this theorem is complete.

\section{Attractivity}

In this section, we discuss the attractivity and uniqueness of time periodic solutions to the problem (1.1)-(1.3).

Theorem 3. Suppose that $\gamma$ satisfies

$$
\bar{b}^{2} / \gamma^{2}+2 \bar{d} / \gamma<\pi^{4}
$$

where $\bar{b}$ and $\bar{d}$ are the upper bounds of $b(t)$ and $d(t)$, respectively. Then the problem (1.1)-(1.3) admits a unique nontrivial periodic solution which attracts all solutions of the initial boundary value problem (1.1), (1.2) and (1.4).

Proof. By virtue of Theorem 1, the problem (1.1)-(1.3) admits a periodic solution $\tilde{u}(x, t)$. Let $u(x, t)$ be any solution of the initial boundary value problem (1.1), (1.2) and (1.4). Define

$$
G(t)=\frac{1}{2} \int_{0}^{1}\left[(u-\tilde{u})^{2}+k|D(u-\tilde{u})|^{2}\right] d x .
$$

By (1.1) and (1.2), we have

$$
\begin{aligned}
G^{\prime}(t) & =\int_{0}^{1}\left[(u-\tilde{u})(u-\tilde{u})_{t}+k D(u-\tilde{u}) D(u-\tilde{u})_{t}\right] d x \\
& =\int_{0}^{1}\left[(u-\tilde{u})_{t}-k D^{2}(u-\tilde{u})_{t}\right](u-\tilde{u}) d x
\end{aligned}
$$




$$
\begin{aligned}
= & \int_{0}^{1}\left[-\gamma D^{4}(u-\tilde{u})+D(\Phi(D u, t)-\Phi(D \tilde{u}, t))\right. \\
& -(A(u, t)-A(\tilde{u}, t))](u-\tilde{u}) d x \\
= & -\gamma \int_{0}^{1}\left|D^{2}(u-\tilde{u})\right|^{2} d x-\int_{0}^{1}(\Phi(D u, t)-\Phi(D \tilde{u}, t))(D u-D \tilde{u}) d x \\
& -\int_{0}^{1}(A(u, t)-A(\tilde{u}, t))(u-\tilde{u}) d x \\
= & -\gamma \int_{0}^{1}\left|D^{2}(u-\tilde{u})\right|^{2} d x+\int_{0}^{1} b(t)|D(u-\tilde{u})|^{2} d x \\
& -\int_{0}^{1} a(t)\left(|D u|^{\alpha-1} D u-|D \tilde{u}|^{\alpha-1} D \tilde{u}\right)(D u-D \tilde{u}) d x \\
& -\int_{0}^{1} c(t)\left(|u|^{\beta-1} u-|\tilde{u}|^{\beta-1} \tilde{u}\right)(u-\tilde{u}) d x+\int_{0}^{1} d(t)(u-\tilde{u})^{2} d x \\
\leq & -\gamma \int_{0}^{1}\left|D^{2}(u-\tilde{u})\right|^{2} d x+\bar{b} \int_{0}^{1}|D(u-\tilde{u})|^{2} d x+\bar{d} \int_{0}^{1}(u-\tilde{u})^{2} d x \\
\leq & -\frac{\gamma}{2} \int_{0}^{1}\left|D^{2}(u-\tilde{u})\right|^{2} d x+\left(\frac{\bar{b}^{2}}{2 \gamma}+\bar{d}\right) \int_{0}^{1}|u-\tilde{u}|^{2} d x .
\end{aligned}
$$

Noticing that the first eigenvalue of the Laplacian equation with homogeneous Dirichlet boundary value conditions in $(0,1)$ is $\pi^{2}$, then

$$
\int_{0}^{1}|D v|^{2} d x \geq \pi^{2} \int_{0}^{1} v^{2} d x, \quad \forall v \in H_{0}^{1}(0,1)
$$

from which we have

$$
\int_{0}^{1}|D(u-\tilde{u})|^{2} d x \geq \rho \int_{0}^{1}|D(u-\tilde{u})|^{2} d x+(1-\rho) \pi^{2} \int_{0}^{1}(u-\tilde{u})^{2} d x
$$

where $\rho \in(0,1)$ is a constant to be determined. Furthermore, by (4.1) and the Hölder inequality, we can deduce that

$$
\int_{0}^{1}\left|D^{2}(u-\tilde{u})\right|^{2} d x \geq \pi^{2} \int_{0}^{1}|D(u-\tilde{u})|^{2} d x .
$$

Consequently,

$G^{\prime}(t) \leq-\frac{\pi^{2} \gamma}{2} \rho \int_{0}^{1}|D(u-\tilde{u})|^{2} d x-\left(\frac{\pi^{4} \gamma}{2}(1-\rho)-\left(\frac{\bar{b}^{2}}{2 \gamma}+\bar{d}\right)\right) \int_{0}^{1}(u-\tilde{u})^{2} d x$

Take $\rho=\frac{\pi^{4} \gamma-\left(\bar{b}^{2} / \gamma+2 \bar{d}\right)}{\left(1 / k+\pi^{2}\right) \pi^{2} \gamma}$, then $0<\rho<1$ and $G^{\prime}(t) \leq-C_{0} G(t)$, where $C_{0}=$ $\frac{\pi^{4} \gamma-\left(\bar{b}^{2} / \gamma+2 \bar{d}\right)}{1+\pi^{2} k}>0$. Integrating the above equality, we get

$$
G(t) \leq G(0) e^{-C_{0} t}
$$


Recalling the definition of $G(t)$, we obtain

$$
\lim _{t \rightarrow \infty}\|u-\tilde{u}\|_{H^{1}(0,1)}=0 .
$$

Hence the time periodic solution $\tilde{u}(x, t)$ attracts all solutions of the initial boundary value problem (1.1), (1.2) and (1.4).

Especially, in the above arguments, let $u(x, t)$ be any time periodic solution of the problem (1.1)-(1.3). Then, for any positive integer $n$, we have

$\|u(x, t)-\tilde{u}(x, t)\|_{H^{1}(0,1)}=\|u(x, t+n \omega)-\tilde{u}(x, t+n \omega)\|_{H^{1}(0,1)} \longrightarrow 0 \quad$ as $n \rightarrow \infty$,

which means that $u(x, t)=\tilde{u}(x, t)$ a.e. in $[0,1] \times[0, T]$. Thus the proof of this theorem is complete.

\section{The Limiting Process as $k$ Tends to Zero}

In this section, we discuss the limiting process of solutions as the viscous coefficient $k$ tends to zero. Throughout this section, we denote by $C$ a constant independent of $u$ and $k$.

Theorem 4. If $u_{k}$ is a time periodic solution of the problem (1.1)-(1.3), then $u_{k}(x, t)$ is uniformly convergent in $Q_{\omega}$ as $k \rightarrow 0$, and the limit function $u(x, t)$ is a time periodic solution of the equation

$$
\frac{\partial u}{\partial t}+\gamma D^{4} u=D \Phi(D u, t)-A(u, t)+f(x, t), \quad(x, t) \in Q_{\omega}
$$

subject to the boundary value conditions (1.2) and periodic condition (1.3).

Proof. Multiplying the equation (1.1) by $u_{k}$ and integrating over $Q_{\omega}$, we have

$$
\begin{gathered}
\gamma \iint_{Q_{\omega}}\left|D^{2} u_{k}\right|^{2} d x d t+\iint_{Q_{\omega}} a(t)\left|D u_{k}\right|^{\alpha+1} d x d t+\iint_{Q_{\omega}} c(t)\left|u_{k}\right|^{\beta+1} d x d t \\
=\iint_{Q_{\omega}} b(t)\left|D u_{k}\right|^{2} d x d t+\iint_{Q_{\omega}} d(t) u_{k}^{2} d x d t+\iint_{Q_{\omega}} f(x, t) u_{k} d x d t \\
\leq \frac{a}{2} \iint_{Q_{\omega}}\left|D u_{k}\right|^{\alpha+1} d x d t+\frac{c}{2} \iint_{Q_{\omega}}\left|u_{k}\right|^{\beta+1} d x d t+C,
\end{gathered}
$$

where $\underline{a}$ and $\underline{c}$ are the lower bounds of $a(t)$ and $c(t)$, respectively. It follows that

$$
\begin{gathered}
\iint_{Q_{\omega}}\left|D^{2} u_{k}\right|^{2} d x d t \leq C, \\
\iint_{Q_{\omega}}\left|D u_{k}\right|^{\alpha+1} d x d t \leq C, \\
\iint_{Q_{\omega}}\left|u_{k}\right|^{\beta+1} d x d t \leq C .
\end{gathered}
$$


We multiply $(1.1)$ by $\frac{\partial u_{k}}{\partial t}$, integrate with respect to $x$ over $(0,1)$ and obtain

$$
\begin{aligned}
& \frac{1}{2} \int_{0}^{1}\left|\frac{\partial u_{k}}{\partial t}\right|^{2} d x+k \int_{0}^{1}\left|\frac{\partial D u_{k}}{\partial t}\right|^{2} d x \\
& \quad+\frac{\mathrm{d}}{\mathrm{d} t} \int_{0}^{1}\left(\frac{\gamma}{2}\left|D^{2} u_{k}\right|^{2}+\Psi\left(D u_{k}, t\right)+B\left(u_{k}, t\right)\right) d x \\
& \leq \int_{0}^{1}\left(\frac{a^{\prime}(t)}{\alpha+1}\left|D u_{k}\right|^{\alpha+1}-\frac{b^{\prime}(t)}{2}\left|D u_{k}\right|^{2}+\frac{c^{\prime}(t)}{\beta+1}\left|u_{k}\right|^{\beta+1}-\frac{d^{\prime}(t)}{2} u_{k}^{2}\right) d x+C,
\end{aligned}
$$

where $\Psi(s, t)$ and $B(s, t)$ are as in (2.5). By (5.3) and (5.4), we have

$$
\iint_{Q_{\omega}}\left|\frac{\partial u_{k}}{\partial t}\right|^{2} d x d t \leq C
$$

Set

$$
F_{k}(t)=\int_{0}^{1}\left(\frac{\gamma}{2}\left|D^{2} u_{k}\right|^{2}+\Psi\left(D u_{k}, t\right)+B\left(u_{k}, t\right)\right) d x, \quad \forall t \in[0, \omega] .
$$

Then, we have

$$
F_{k}^{\prime}(t) \leq C F_{k}(t)+C, \quad \forall t \in[0, \omega] .
$$

Similar to the proof of Theorem 1 , we obtain $F_{k}(t) \leq C, \forall t \in[0, \omega]$, from which we have

$$
\int_{0}^{1}\left|D^{2} u_{k}(x, t)\right|^{2} d x \leq C, \quad \int_{0}^{1}\left|D u_{k}(x, t)\right|^{\alpha+1} d x \leq C, \quad \forall t \in[0, \omega] .
$$

By (5.6), we arrive at

$$
\left\|u_{k}\right\|_{L^{\infty}\left(\bar{Q}_{\omega}\right)} \leq C, \quad\left\|D u_{k}\right\|_{L^{\infty}\left(\bar{Q}_{\omega}\right)} \leq C .
$$

Multiplying (1.1) by $D^{4} u_{k}$ and integrating over $Q_{\omega}$ give

$$
\iint_{Q_{\omega}}\left|D^{4} u_{k}\right|^{2} d x d t \leq C
$$

Rewrite the equation (1.1) into the following form

$$
k \frac{\partial D^{2} u_{k}}{\partial t}=\frac{\partial u_{k}}{\partial t}+\gamma D^{4} u_{k}-\Phi_{s}\left(D u_{k}, t\right) D^{2} u_{k}+A\left(u_{k}, t\right)-f(x, t),
$$

where $\Phi_{s}^{\prime}(x, t)=a(t) \alpha|s|^{\alpha-1}-b(t)$. From (5.2), (5.5), (5.7) and (5.8), we get

$$
k \iint_{Q_{\omega}}\left|\frac{\partial D^{2} u_{k}}{\partial t}\right|^{2} d x d t \leq C .
$$

Similar to the proof of Theorem 1, we have

$$
\left|u_{k}\left(x_{1}, t_{1}\right)-u_{k}\left(x_{2}, t_{2}\right)\right| \leq C\left(\left|x_{1}-x_{2}\right|+\left|t_{1}-t_{2}\right|^{1 / 4}\right)
$$


for all $\left(x_{i}, t_{i}\right) \in \bar{Q}_{\omega}(i=1,2)$. From (1.1), we see that $v_{k}=D u_{k}$ satisfies

$$
\frac{\partial v_{k}}{\partial t}+\gamma D^{4} v_{k}-k \frac{\partial D^{2} v_{k}}{\partial t}=D^{2} \Phi\left(v_{k}, t\right)-D A\left(u_{k}, t\right)+D f(x, t) .
$$

Similarly, we can deduce that

$$
\left|D u_{k}\left(x_{1}, t_{1}\right)-D u_{k}\left(x_{2}, t_{2}\right)\right| \leq C\left(\left|x_{1}-x_{2}\right|^{1 / 2}+\left|t_{1}-t_{2}\right|^{1 / 8}\right)
$$

for all $\left(x_{i}, t_{i}\right) \in \bar{Q}_{\omega}(i=1,2)$.

Recalling the equation (1.1), for any $\varphi \in C^{2}\left(\bar{Q}_{\omega}\right)$ satisfying $\varphi(0, t)=$ $\varphi(1, t)=0$ and $\varphi(x, \omega)=\varphi(x, 0)$, we have

$$
\begin{aligned}
& \iint_{Q_{\omega}} \frac{\partial u_{k}}{\partial t} \varphi d x d t+\gamma \iint_{Q_{\omega}} D^{4} u_{k} \varphi d x d t-k \iint_{Q_{\omega}} \frac{\partial u_{k}}{\partial t} D^{2} \varphi d x d t \\
& \quad-\iint_{Q_{\omega}} \alpha a(t)\left|D u_{k}\right|^{\alpha-1} D^{2} u_{k} \varphi d x d t+\iint_{Q_{\omega}} b(t) D^{2} u_{k} \varphi d x d t \\
& \quad+\iint_{Q_{\omega}} c(t)\left|u_{k}\right|^{\beta-1} u_{k} \varphi d x d t-\iint_{Q_{\omega}} d(t) u_{k} \varphi d x d t=\iint_{Q_{\omega}} f(x, t) \varphi d x d t .
\end{aligned}
$$

From (5.2)-(5.10), there exists a function $u \in H^{4,1}\left(Q_{\omega}\right) \cap C^{1+\mu, \mu / 4}\left(\bar{Q}_{\omega}\right)$ with $\mu \in(0,1 / 2)$ such that

$$
\begin{aligned}
& u_{k} \longrightarrow u \quad \text { uniformly in } Q_{\omega}, \\
& D u_{k} \longrightarrow D u \quad \text { in } C^{\mu, \mu / 4}\left(Q_{\omega}\right) \text { for any } 0<\mu<1 / 2, \\
& D^{2} u_{k} \rightarrow D^{2} u, \quad D^{4} u_{k} \rightarrow D^{4} u, \quad \frac{\partial u_{k}}{\partial t} \rightarrow \frac{\partial u}{\partial t} \quad \text { in } L^{2}\left(Q_{\omega}\right),
\end{aligned}
$$

as $k \rightarrow 0$. Therefore, taking $k \rightarrow 0$ we have

$$
\begin{aligned}
& \iint_{Q_{\omega}} \frac{\partial u}{\partial t} \varphi d x d t+\gamma \iint_{Q_{\omega}} D^{4} u \varphi d x d t \\
& \quad-\iint_{Q_{\omega}} \alpha a(t)|D u|^{\alpha-1} D^{2} u \varphi d x d t+\iint_{Q_{\omega}} b(t) D^{2} u \varphi d x d t \\
& \quad+\iint_{Q_{\omega}} c(t)|u|^{\beta-1} u \varphi d x d t-\iint_{Q_{\omega}} d(t) u \varphi d x d t=\iint_{Q_{\omega}} f(x, t) \varphi d x d t
\end{aligned}
$$

that is

$$
\begin{aligned}
& \iint_{Q_{\omega}} \frac{\partial u}{\partial t} \varphi d x d t+\gamma \iint_{Q_{\omega}} D^{4} u \varphi d x d t \\
& \quad-\iint_{Q_{\omega}} D \Phi(D u, t) \varphi d x d t+\iint_{Q_{\omega}} A(u, t) \varphi d x d t=\iint_{Q_{\omega}} f(x, t) \varphi d x d t .
\end{aligned}
$$

Hence $u \in H^{4,1}\left(Q_{\omega}\right) \cap C^{1+\mu, \mu / 4}\left(\bar{Q}_{\omega}\right)$ satisfies the equation (5.1) in the sense of distribution. Moreover, the equation (5.1) is equivalent to

$$
\frac{\partial u}{\partial t}+\gamma D^{4} u=\Phi_{s}^{\prime}(D u, t) D^{2} u-A(u, t)+f(x, t), \quad(x, t) \in Q_{\omega}
$$


where $\Phi_{s}^{\prime}(D u, t)=\alpha a(t)|D u|^{\alpha-1}-b(t)$. From $u \in C^{1+\mu, \mu / 4}\left(\bar{Q}_{\omega}\right), \alpha \geq 2$ we know that $\Phi_{s}^{\prime}(D u, t) \in C^{\mu, \mu / 4}\left(\bar{Q}_{\omega}\right)$. By the classical linear theory (see [9]), we have $u \in C^{4+\mu, 1+\mu / 4}\left(\bar{Q}_{\omega}\right)$ with $\mu \in(0,1 / 2)$. Obviously, the solution $u$ satisfies the boundary value conditions (1.2) and the periodic condition (1.3). Therefore, $u$ is a time periodic solution of the problem (5.1), (1.2) and (1.3). The proof is complete.

At last, we study the limit process of solutions to the initial boundary value problem (1.1), (1.2) and (1.4) as $k \rightarrow 0$.

Theorem 5. If $u_{k}$ is a solution of the initial boundary value problem (1.1), (1.2) and (1.4), then $u_{k}(x, t)$ is uniformly convergent in $Q_{T}$ as $k \rightarrow 0$, and the limit function $u(x, t)$ is a solution of the equation

$$
\frac{\partial u}{\partial t}+\gamma D^{4} u=D \Phi(D u, t)-A(u, t)+f(x, t), \quad(x, t) \in Q_{T}
$$

subject to the initial boundary value conditions (1.2) and (1.4).

Proof. Multiplying (1.1) with $u_{k}$ and integrating over $Q_{t}$, we have

$$
\begin{aligned}
& \frac{1}{2} \int_{0}^{1} u_{k}^{2}(x, t) d x+\gamma \iint_{Q_{t}}\left|D^{2} u_{k}\right|^{2} d x d \tau+\frac{k}{2} \int_{0}^{1}\left|D u_{k}(x, t)\right|^{2} d x \\
&+\iint_{Q_{t}} a(t)\left|D u_{k}\right|^{\alpha+1} d x d \tau+\iint_{Q_{t}} c(t)\left|u_{k}\right|^{\beta+1} d x d \tau \\
&= \frac{1}{2} \int_{0}^{1} u_{k 0}^{2}(x) d x+\frac{k}{2} \int_{0}^{1}\left|D u_{k 0}(x)\right|^{2} d x \\
& \quad+\iint_{Q_{t}} b(t)\left|D u_{k}\right|^{2} d x d \tau+\iint_{Q_{t}} d(t) u_{k}^{2} d x d \tau+\iint_{Q_{t}} f(x, t) u_{k} d x d \tau \\
& \quad \leq \frac{a}{2} \iint_{Q_{t}}\left|D u_{k}\right|^{\alpha+1} d x d \tau+\frac{c}{2} \iint_{Q_{t}}\left|u_{k}\right|^{\beta+1} d x d \tau+C+C k,
\end{aligned}
$$

where $\underline{a}$ and $\underline{c}$ are the lower bounds of $a(t)$ and $c(t)$, respectively, from which we have

$$
\begin{aligned}
& \iint_{Q_{T}}\left|D^{2} u_{k}\right|^{2} d x d t \leq C+C k \\
& \iint_{Q_{T}}\left|D u_{k}\right|^{\alpha+1} d x d t \leq C+C k, \quad \iint_{Q_{T}}\left|u_{k}\right|^{\beta+1} d x d t \leq C+C k
\end{aligned}
$$

We multiply (1.1) by $\frac{\partial u_{k}}{\partial t}$, integrate the result over $Q_{t}$ and obtain

$$
\begin{aligned}
& \frac{1}{2} \iint_{Q_{t}}\left|\frac{\partial u_{k}}{\partial \tau}\right|^{2} d x d \tau+k \iint_{Q_{t}}\left|\frac{\partial D u_{k}}{\partial \tau}\right|^{2} d x d \tau+\frac{\gamma}{2} \int_{0}^{1}\left|D^{2} u_{k}(x, t)\right|^{2} d x \\
& \quad+\int_{0}^{1} \Psi\left(D u_{k}(x, t), t\right) d x+\int_{0}^{1} B\left(u_{k}(x, t), t\right) d x \leq C+C k,
\end{aligned}
$$


where $\Psi(s, t)$ and $B(s, t)$ are as in (2.5). By using Young's inequality, we get

$$
\begin{aligned}
& \iint_{Q_{t}}\left|\frac{\partial u_{k}}{\partial \tau}\right|^{2} d x d \tau+2 k \iint_{Q_{t}}\left|\frac{\partial D u_{k}}{\partial \tau}\right|^{2} d x d \tau+\gamma \int_{0}^{1}\left|D^{2} u_{k}(x, t)\right|^{2} d x \\
& \quad+\frac{\underline{a}}{\alpha+1} \int_{0}^{1}\left|D u_{k}(x, t)\right|^{\alpha+1} d x+\frac{\underline{c}}{\beta+1} \int_{0}^{1}\left|u_{k}(x, t)\right|^{\beta+1} d x \leq C+C k .
\end{aligned}
$$

It follows that

$$
\begin{gathered}
\iint_{Q_{T}}\left|\frac{\partial u_{k}}{\partial t}\right|^{2} d x d t \leq C+C k, \\
\int_{0}^{1}\left|D^{2} u_{k}(x, t)\right|^{2} d x \leq C+C k, \quad \forall t \in(0, T), \\
\int_{0}^{1}\left|D u_{k}(x, t)\right|^{\alpha+1} d x \leq C+C k, \quad \forall t \in(0, T) .
\end{gathered}
$$

From (5.15) and (5.16), we deduce that

$$
\left\|u_{k}\right\|_{L^{\infty}\left(\bar{Q}_{T}\right)} \leq C+C k, \quad\left\|D u_{k}\right\|_{L^{\infty}\left(\bar{Q}_{T}\right)} \leq C+C k .
$$

Multiplying (1.1) by $D^{4} u_{k}$ and integrating over $Q_{T}$, we get

$$
\iint_{Q_{T}}\left|D^{4} u_{k}\right|^{2} d x d t \leq C+C k .
$$

By virtue of the equation (1.1), we obtain

$$
k \iint_{Q_{T}}\left|\frac{\partial D^{2} u_{k}}{\partial t}\right|^{2} d x d t \leq C+C k .
$$

Using the above a priori estimates, we arrive at

$$
\begin{aligned}
\left|u_{k}\left(x_{1}, t_{1}\right)-u_{k}\left(x_{2}, t_{2}\right)\right| & \leq(C+C k)\left(\left|x_{1}-x_{2}\right|+\left|t_{1}-t_{2}\right|^{1 / 4}\right), \\
\left|D u_{k}\left(x_{1}, t_{1}\right)-D u_{k}\left(x_{2}, t_{2}\right)\right| & \leq(C+C k)\left(\left|x_{1}-x_{2}\right|^{1 / 2}+\left|t_{1}-t_{2}\right|^{1 / 8}\right)
\end{aligned}
$$

for all $\left(x_{i}, t_{i}\right) \in \bar{Q}_{T}(i=1,2)$. Hence, there exists a function $u \in H^{4,1}\left(Q_{\omega}\right) \cap$ $C^{1+\mu, \mu / 4}\left(\bar{Q}_{\omega}\right)$ with $\mu \in(0,1 / 2)$. such that

$u_{k} \longrightarrow u$ uniformly in $Q_{T}$,

$D u_{k} \longrightarrow D u \quad$ in $C^{\mu, \mu / 4}\left(Q_{T}\right)$ for any $0<\mu<1 / 2$,

$D^{2} u_{k} \rightarrow D^{2} u, \quad D^{4} u_{k} \rightarrow D^{4} u, \quad \frac{\partial u_{k}}{\partial t} \rightarrow \frac{\partial u}{\partial t} \quad$ in $L^{2}\left(Q_{T}\right)$,

as $k \rightarrow 0$. Similar to the discussion in Theorem 4, we can prove that $u$ satisfies the equation (5.11) in the sense of distribution. We rewrite the equation (5.11) into the following form

$$
\frac{\partial u}{\partial t}+\gamma D^{4} u=\Phi_{s}^{\prime}(D u, t) D^{2} u-A(u, t)+f(x, t), \quad(x, t) \in Q_{T} .
$$


Noticing that $\Phi_{s}^{\prime}(D u, t) \in C^{\mu, \mu / 4}\left(\bar{Q}_{\omega}\right)$, by the classical linear theory (see [9]) we see that $u \in C^{4+\mu, 1+\mu / 4}\left(\bar{Q}_{\omega}\right)$ with $\mu \in(0,1 / 2)$. Moreover, it is obviously that $u$ satisfies the conditions (1.2) and (1.4). Therefore, $u$ is a solution of the initial boundary value problem $(5.11),(1.2)$ and (1.4). The proof of this theorem is complete.

\section{Acknowledgments}

This work is supported by 973 Program (2010CB808002), NNSFC, CPSF, SRFDP and the Fundamental Research Funds for the Central Universities. The authors would like to express their sincere thanks to their supervisor Prof. Yin Jingxue in South China Normal University for his kind guidance. The authors would like to thank the referees for their valuable comments.

\section{References}

[1] F. Bai, C.M. Elliott, A. Gardiner, A. Spence and A.M. Stuart. The viscous Cahn-Hilliard equation. I: Computations. Nonlinearity, 8(2):131-160, 1995. http://dx.doi.org/10.1088/0951-7715/8/2/002.

[2] J. Cao, A. Chen and X. Huang. Almost periodic attractor of delayed neural networks with variable coefficients. Phys. Lett. A, 340(1-4):104-120, 2005. http://dx.doi.org/10.1016/j.physleta.2005.04.021.

[3] R. Chill, E. Fas̆angová and J. Prüss. Convergence to steady states of solutions of the Cahn-Hilliard and caginalp equations with dynamic boundary conditions. Math. Nachr., 279(13-14):1448-1462, 2006. http://dx.doi.org/10.1002/mana.200410431.

[4] C.M. Elliott and A.M. Stuart. Viscous Cahn-Hilliard equation. II. Analysis. J. Differential Equations, 128(2):387-414, 1996. http://dx.doi.org/10.1006/jdeq.1996.0101.

[5] C.M. Elliott and S.M. Zheng. On the Cahn-Hilliard equation. Arch. Ration. Mech. Anal., 96(4):339-357, 1986. http://dx.doi.org/10.1007/BF00251803.

[6] Y. He and Y. Liu. Stability and convergence of the spectral Galerkin method for the Cahn-Hilliard equation. Numer. Methods Partial Differential Equations, 24(6):1485-1500, 2008. http://dx.doi.org/10.1002/num.20328.

[7] B.B. King, O. Stein and M. Winkler. A fourth-order parabolic equation modeling epitaxial thin film growth. J. Math. Anal. Appl., 286(2):459-490, 2003. http://dx.doi.org/10.1016/S0022-247X(03)00474-8.

[8] I. Kubiaczyk and S.H. Saker. Oscillation and stability in nonlinear delay differential equations of population dynamics. Math. Comput. Modelling, 35(34):295-301, 2002. http://dx.doi.org/10.1016/S0895-7177(01)00166-2.

[9] O. Ladyzenskaja, V. Solonnikov and N. Uraltseva. Linear and Quasilinear Equations of Parabolic Type. American Mathematical Society, Providence, 1968.

[10] R. Laugesen and M. Pugh. Linear stability of steady states for thin film and Cahn-Hilliard type equations. Arch. Ration. Mech. Anal., 154(1):3-51, 2000. http://dx.doi.org/10.1007/PL00004234.

[11] Y. Li and J. Yin. The viscous Cahn-Hilliard equation with periodic potentials and sources. J. Fixed Point Theory Appl., 9(1):63-84, 2011. http://dx.doi.org/10.1007/s11784-010-0014-z. 
[12] Y. Li, J. Yin and C. Jin. A Cahn-Hilliard type equation with periodic gradient dependent potentials and sources. Math. Methods Appl. Sci., 33(3):263-272, 2010. http://dx.doi.org/10.1002/mma.1166.

[13] C. Liu. A fourth order parabolic equation with nonlinear principal part. Nonlinear Anal., 68(2):393-401, 2008. http://dx.doi.org/10.1016/j.na.2006.11.005.

[14] S. Liu, F. Wang and H. Zhao. Global existence and asymptotic of solutions of the Cahn-Hilliard equation. J. Differential Equations, 238(2):426-469, 2007. http://dx.doi.org/10.1016/j.jde.2007.02.014.

[15] A. Miranville and A. Rougirel. Local and asymptotic analysis of the flow generated by the Cahn-Hilliard-Gurtin equations. Z. Angew. Math. Phys., 57(2):244268, 2006. http://dx.doi.org/10.1007/s00033-005-0017-6.

[16] J.D. Murray. Mathematical Biology, second ed. Springer-Verlag, Berlin, 1993.

[17] B. Nicolaenko, B. Scheurer and R. Temam. Some global dynamical properties of a class of pattern formation equations. Comm. Partial Differential Equations, 14(2):245-297, 1989. http://dx.doi.org/10.1080/03605308908820597.

[18] A. Novick-Cohen. On the viscous Cahn-Hilliard equation. In Material Instabilities in Continuum Mechanics, pp. 329-342, New York, 1988. Oxford Univ. Press.

[19] C. V. Pao. Stability and attractivity of periodic solutions of parabolic systems with time delays. J. Math. Anal. Appl., 304(2):423-450, 2005. http://dx.doi.org/10.1016/j.jmaa.2004.09.014.

[20] P. Rybka and K. H. Hoffmann. Convergence of solutions to Cahn-Hilliard equation. Comm. Partial Differential Equations, 24(5-6):1055-1077, 1999. http://dx.doi.org/10.1080/03605309908821458.

[21] J. Yin, Y. Li and R. Huang. The Cahn-Hilliard type equations with periodic potentials and sources. Appl. Math. Comput., 211(1):211-221, 2009. http://dx.doi.org/10.1016/j.amc.2009.01.038.

[22] J. Yin and Y. Wang. Asymptotic behavior of solutions for porous medium equation with periodic absorption. Int. J. Math. Math. Sci., 26(1):35-44, 2001. http://dx.doi.org/10.1155/S0161171201003581.

[23] L. Yin, Y. Li, R. Huang and J. Yin. Time periodic solutions for a Cahn-Hilliard type equation. Math. Comput. Modelling, 48(1):11-18, 2008. http://dx.doi.org/10.1016/j.mcm.2007.09.001. 